\title{
Vies dans le Haut-Tessin traditionnel
}

\author{
Jean Martin
}

Dr méd., membre de la rédaction

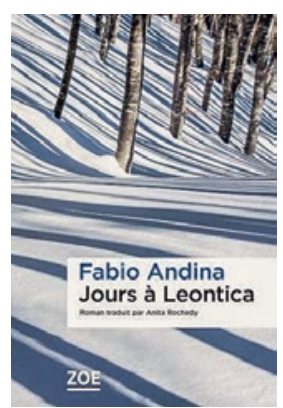

Fabio Andina Jours à Leontica

Editions Zoé: Chêne-Bourg/Genève; 2021, 252 pages
C'est un livre coloré, fascinant, que celui de Fabio Andina, écrivain tessinois né en 1972. Il est nommé roman mais, à le parcourir, on a l'impression forte de circonstances vécues, ce qui pousse à le qualifier de récit documentaire. L'auteur a été enfant à Leontica, dans le val Blenio, au-dessus d'Acquarossa, et connaît bien la nature, la vie et la communauté qu'il raconte.

Fabio Andina nous offre la description frugale, sans précipitation, d'une microsociété, d'une microculture. Le héros en est «le Felice», vieux garçon marié une fois durant quelques mois, qui a, comme jeune adulte, passé quelque temps en Russie dans les années 1960. A part cet épisode, il a toujours vécu au village. Le narrateurqu'on imagine proche de l'auteur - vit dans un chalet voisin de celui de Felice et l'accompagne dans ses tournées et visites dans cette microrégion. Felice a 90 ans et vit nu-pieds durant l'essentiel de l'année; en saison froide, il porte ses vieilles chaussures militaires. Son rite est de se lever chaque matin vers 5 heures et de monter durant plus d'une heure dans la montagne pour prendre un bain dans un bassin naturel glacial. Le narrateur l'accompagne. La montée est ponctuée d'arrêts réguliers, notamment à l'étable du Sosto, où on bénéficie d'un verre de grappa au retour. On dérange parfois des cerfs.

Je suis issu d'un milieu de paysans et vignerons du Plateau vaudois, mes ascendants avaient, mutatis mutandis, des habitudes de vie proches de celles des gens de Leontica quant à la simplicité du quotidien.

Fabio Andina offre la description frugale d'une microsociété, d'une microculture. Le héros en est «le Felice», vieux garçon de 90 ans.

Dans mon enfance, on disait «Le Paul», «La Juliette». Cela est passé de mode, mais c'est encore la règle à Leontica, ce qui peut parfois déboussoler le lecteur par les mentions mêlées des noms des personnes et des animaux domestiques. A la lecture, on se demande quelle époque est décrite. Il s'agit pourtant du début du XXI ${ }^{\mathrm{e}}$ siècle: presque chaque maison a un frigo, certains ont un véhicule utilitaire, un portable, une adresse internet. Au bar Gallo Cedrone, la serveuse interrompt les conversations parce que, à la télé, la skieuse Lara Gut est en course. Les 250 pages regorgent de personnages pittoresques, hommes et femmes, souvent au-delà des 80 ans, quelques enfants aussi, dont on apprend les habitudes. Sont dépeints des tableaux de la vie quotidienne: la préparation des repas qui prend du temps, le défilé de la Milice historique tous les 24 juin, pour la Saint-Jean. Faits et gestes, discussions ou moments taciturnes, rencontres, tout se déroule calmement. Pas de rognes éternelles, de «bringues» de famille ou de voisinage. Est-ce vraiment possible? Tout le monde n'aime pas forcément tout le monde, mais on se côtoie, on se parle. Je ne dévoile pas la clé de l'énigme de l'installation chez le Felice d'un deuxième lit dans une chambre vide. Sa femme reviendrait-elle, après des décennies?

\section{Ce roman a été soutenu dans un sens d'inter-} connaissance et de solidarité confédérales. La vie décrite pourrait ne plus exister longtemps.

L'apothéose, c'est la découverte de Felice mort dans son lit, un matin où comme de coutume le narrateur vient le chercher pour monter à la "pozza». Tout le village débarque peu à peu: le médecin d'Acquarossa qui constate le décès, le curé qui est éconduit parce qu'on sait que le Felice n'aurait pas voulu d'un service religieux, le maire. Chacune et chacun vient avec boissons et victuailles, on ouvre des bouteilles, un grand repas est préparé. Sans excès, l'ambiance s'anime. La stupéfaction c'est quand, quelques heures après que le Pep, ami proche du disparu, a fait un mystérieux téléphone, arrive un fourgon zurichois. La communauté découvre que le Felice a donné son corps à la science, corps emmené après que les convoyeurs ont partagé une tasse de café et accepté un casse-croûte. Coup de théâtre posthume du vieux solitaire.

Ce récit a bénéficié d'aides à la culture dans un sens d'interconnaissance et de solidarité confédérales. Une excellente initiative pour cette description d'une vie dont il est probable qu'elle ne se maintiendra pas longtemps telle quelle, sur sa montagne dans la vallée du Lukmanier. Maintenant déjà, nos enfants vivant pour l'essentiel en milieu urbain ne peuvent guère imaginer ce que raconte Jours à Leontica, tout près de nous pourtant. Je crois que l'essentiel y est véridique mais, de toute manière et par hypothèse, se non è vero, è ben trovato. 